\title{
Navigation for Indoor Robot: Straight Line Movement via Navigator
}

\author{
Chaozheng Zhu ${ }^{D},{ }^{1}$ Ming He $\left(\mathbb{D},{ }^{1,2}\right.$ Pan Chen, ${ }^{3}$ Kang Sun, \\ Jinglei Wang $\mathbb{Q}^{5}$ and Qian Huang ${ }^{3,6}$ \\ ${ }^{1}$ College of Command and Control Engineering, The Army Engineering University of PLA, Nanjing 210007, China \\ ${ }^{2}$ Institute of Network Information, Institute of Systems Engineering, Academy of Military Sciences, Beijing 100071, China \\ ${ }^{3}$ College of Computer and Information, Hohai University, Nanjing 211100, China \\ ${ }^{4}$ College of Energy and Electrical Engineering, Hohai University, Nanjing 211100, China \\ ${ }^{5}$ School of Electronic and Optical Engineering, Nanjing University of Science and Technology, Nanjing 210094, China \\ ${ }^{6}$ Key Laboratory of Symbol Computation and Knowledge Engineering of Ministry of Education, Jilin University, Jilin 130012, China
}

Correspondence should be addressed to Ming He; paper_review@126.com

Received 4 July 2018; Accepted 2 September 2018; Published 24 September 2018

Academic Editor: Simone Bianco

Copyright (c) 2018 Chaozheng Zhu et al. This is an open access article distributed under the Creative Commons Attribution License, which permits unrestricted use, distribution, and reproduction in any medium, provided the original work is properly cited.

Due to the need of zigzag overlay strategy, long-term linear motion is essential for sweep robot. However, the existing indoor sweep robot navigation algorithm has many problems; for instance, algorithm with high complexity demands high hardware performance and is incapable of working at night. To overcome those problems, in this paper, a new method for indoor robot Straight Line Movement via Navigator (SLMN) is proposed to ensure long linear motion of robot with an acceptable error threshold and realize multiroom navigation. Firstly, in a short time, robot runs a suitable distance when it is covered by navigator's ultrasonic sensor. We can obtain a triangle with twice the distance between navigator and robot and the distance of robot motion. The forward angle of the robot can be conveniently obtained by the trigonometric functions. Comparing the robot's current angle with expected angle, the robot could correct itself and realize the indoor linear navigation. Secondly, discovering dozens of the magnitude gaps between the distance of robot run and the distance between navigator and robot, we propose an optimized method using approximate scaling which increases efficiency by nearly $70.8 \%$. Finally, to realize multiroom navigation, we introduce the conception of the depth-first search stack and a unique encode rule on rooms and navigators. It is proved by extensive quantitative evaluations that the proposed method realizes indoor full coverage at a lower cost than other state-of-the-art indoor vision navigation schemes, such as ORB-SLAM.

\section{Introduction}

Traditional low cost sweep robot used the random mode of sweeping, with small coverage area, low cleaning efficiency, and easy collision. Adopting the shape of $\mathrm{Z}$ full coverage algorithm [1] can effectively reduce the disadvantage of random sweeping, but robot must run linearly if the full coverage algorithm can work steadily $[2,3]$. It could work normally based on an inertial measurement unit (IMU) [4,5], but with the data from both sensor and PID correcting its direction, it is not enough to realize its indoor localization. Due to accumulated errors of sensors, wheel mechanical inaccuracy, and the slide problem, sweeping robot would drift away, so researchers start the indoor navigation system [6] research.

Rodas et al. proposed an optimal Bayesian fusion and localization algorithm based on Bluetooth positioning data [7]. However, Bluetooth localization error is still serious, deployment cost is huge, and the result is not satisfactory. Lin et al. proposed a fingerprint localization system based on Wi$\mathrm{Fi}[8]$, which is a sample of signal intensity, and preconstruct a radio map, use the information of Wi-Fi cover, and read data from accelerometer reliably. However, a great quantity initial work should be finished before using it, and the effect is not very well. Fuentes-Pacheco et al. proposed vSLAM (visual Simultaneous Localization and Mapping) [9-13]; this scheme 


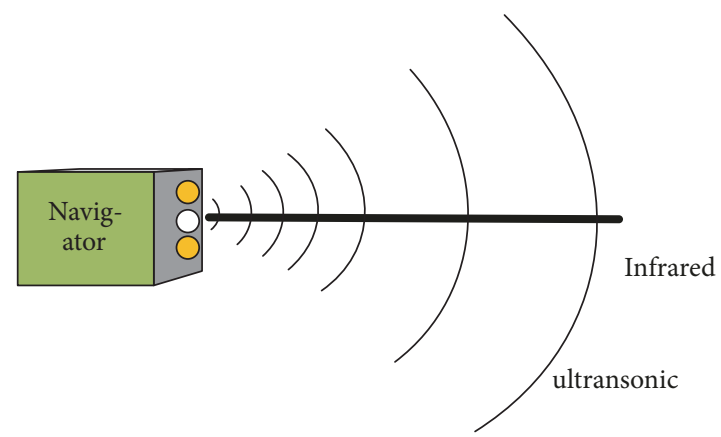

Figure 1: Infrared ray and ultrasonic wave emission diagram of navigator. From theoretical analysis, the analytic method of deviation angle alpha.

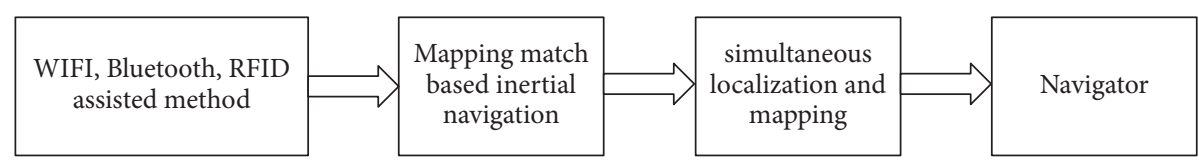

FIGURE 2: The development route of indoor navigation.

has lower deployment cost and high localization accuracy features, and also its visualization is prefect. However, when the light had changed, the result fluctuated greatly. With introducing a Kinect (a depth camera) [14], the cost of full system would rise. In [15], Binu proposed to use the naturally occurring magnetic fields inside the concrete buildings with simplified magnetic map [16] matching algorithm. Due to the existence of sensor accumulation error problems, the navigation's accuracy is insufficient. Furthermore, Wolfgang Hess et al. propose a 2D SLAM system that combines scanto-submap matching with loop closure detection and graph optimization, making its source codes open-source [17]. However, equally it needs a LIDAR, which costs much money.

Because of the characteristics of light moving straight forward, the infrared ray [18] wavelength is long and mostly would not scatter in the air. An infrared ray is sent by opening a small hole in the front of the navigator, so that it can be considered as a line in terms of the ultrasonic (see Figure 1). The infrared communication module consists of transmitter and receiver. The transmitter is located on the navigator, and the receiver is located on the sweeping robot. A low scattering infrared ray is sent by the transmitter, after being received by the sweeping robot, and it can be regarded as a baseline in the room (we usually put the navigator approximately on the middle of the room's edge) [19].

Similarly, the ultrasonic module is divided into the transmitter and the receiver. The transmitter is located on the navigator, and the receiver is located on the sweeping robot. Measuring the time of running between the navigator and the sweeping robot and multiplying by the velocity of ultrasonic in the air, we can work out the distance between them. While meeting the infrared baseline, the sweeping robot would start to correct itself. And because the infrared ray has the reflection characteristic, when the infrared ray radiates to the ceiling, the reflection will occur, so that the full room would be covered [20].
In a word, to realize indoor linear navigation, the deployment cost based on Wi-Fi [21, 22] and Bluetooth is huge, and vSLAM cannot work normally in the evening. Therefore, when considering the cost problem with guaranteeing high coverage percentages, we propose to use a navigator (consisting of infrared and ultrasonic) to help localization and navigation. It mainly carries out the shape of $Z$ strategy based on motor encoder and PID controller local optimum [23]. At the same time, infrared ray and ultrasonic are used to realize auxiliary localization and correct itself to make global optimum [18]. Comparing to existing algorithms, extensive quantitative evaluations shows that the proposed method realizes indoor full coverage at a lower cost than other stateof-the-art indoor vision navigation schemes, such as ORBSLAM $[11,24]$ (see Figure 2).

The remainder of this paper is organized as follows. Section 2 provides an overview of the robot hardware platform. Section 3 explains the proposed localization and navigation algorithm. Finally, Section 4 provides experimental results and comparisons.

\section{Robotic Platform}

In order to verify the navigation algorithm, this paper designs a double navigation platform between sweeping robot and navigator. The sweeping robot adopts Cortex-M3 architecture 32 bit microcontroller stm 32 as the main control chip, which is produced by STMicroelectronics. The navigator adopts super low power msp430 as the main control chip, which is produced by the United States TI. A sweeping robot consists of two wheels controlled by deceleration motors, equipped with photoelectric encoders to measure the speed of the two wheels, and uses six-axis gyroscope MPU6050 to calculate its posture [25]. Additionally, the sweeping robot and navigator are equipped with infrared transceiver module to communicate with each other at the top [20]. In flank, an 


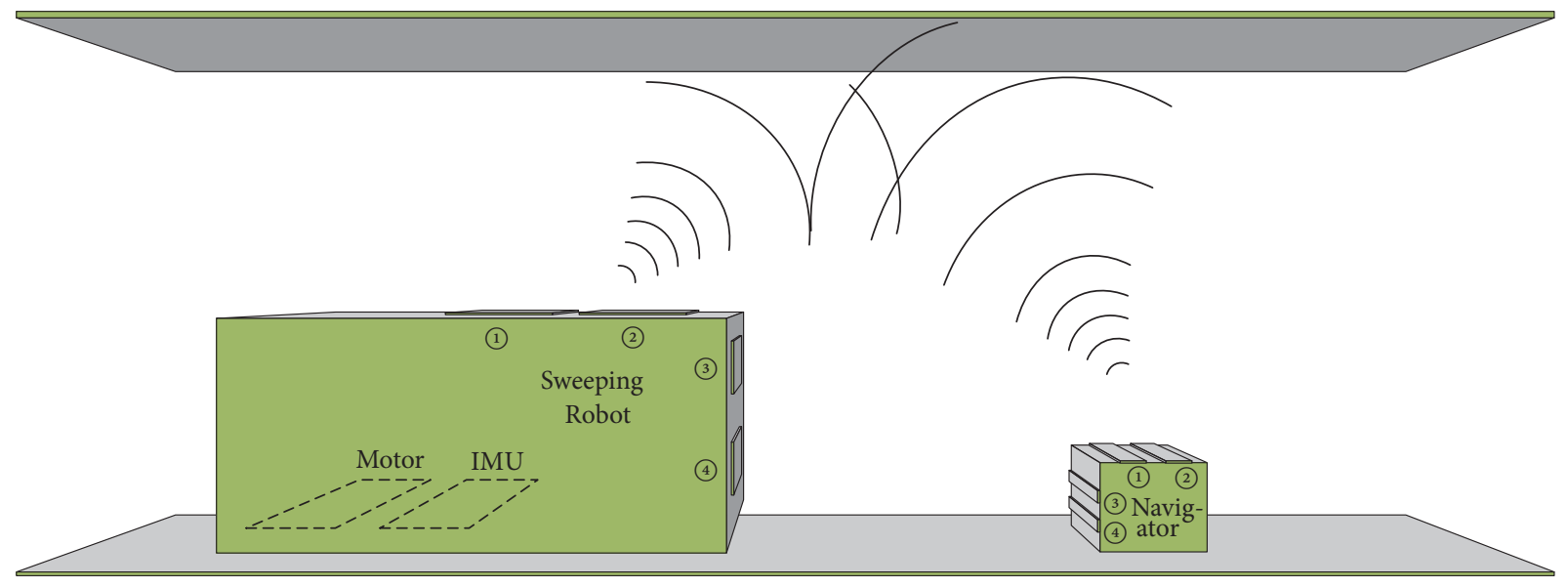
(1) Infrared reception
(3) Ultrasonic reception
(2) Infrared transmission
(4) Ultrasonic transmission

FIGURE 3: The infrared relies on ceiling reflection to cover full room communication.

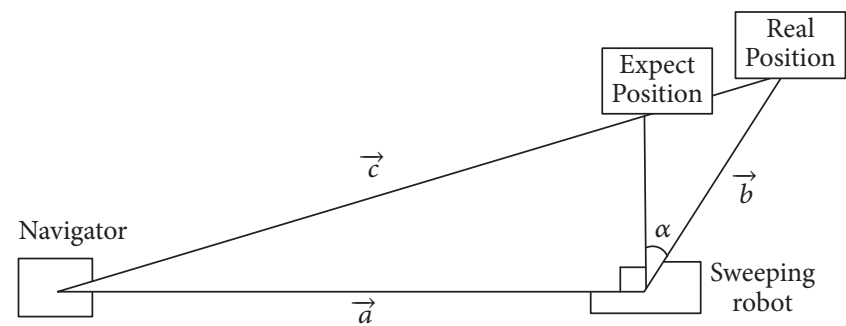

FIgURE 4: From theoretical analysis, the analytic method of deviation angle $\alpha$.

infrared sensor and ultrasonic sensor are used to obtain the distance between them as shown in Figure 3.

\section{Localization and Navigation of Indoor Robot}

The navigator is located at the edge of the room, and it regularly sends an infrared ray signals without scattering. When the sweeping robot detects infrared ray signal, calculates the distance $\vec{a}$, and records the wheels roll over the distance $\vec{b}$, the ultrasonic wave starts to emit regular frequency. With the ultrasonic wave, we could measure $\vec{c}$ again. As a result, by solving $\vec{a}, \vec{b}, \vec{c}$ which form a vector triangle, we can obtain the real forward angle and correct the sweeping robot direction by comparison to the theoretical value as shown in Figure 4.

3.1. Ensure Running in a Straight Line. Traditionally, a sweeping robot can stably execute straight line because two wheels can keep themselves running with the same velocity. Because the electronic characteristics of two wheels may not be completely consistent, in the same PWM duty rate, the velocities of two motors are not exactly the same. If there is long-term running, the error is accumulated continually, and the sweeping robot would move forward one side. Therefore, it is necessity to adjust the velocity of the two wheels in real time so that the velocity of two wheels becomes the same, and robot can keep itself linearly running for a long time without any consideration to the other obstacles.

In this paper, we installed two 26-hole photoelectric tachometer encoders at the back of the motors. By measuring the number of pulses $N$ generated by the photoelectric encoder in unit time $T$ and before that measuring the circumference of the wheel $L$, the formula is given as follows.

$$
V=\frac{(N / 26) * L}{T}
$$

Theoretically, after obtaining the forward speed of $V$, the velocity of the wheel can be adjusted by the PID control algorithm in the field of classical automatic control. However, considering the approximate errors produced on the formula transformation, we directly added the number of pulses $N$ generated by the photoelectric encoder to the PID formula 


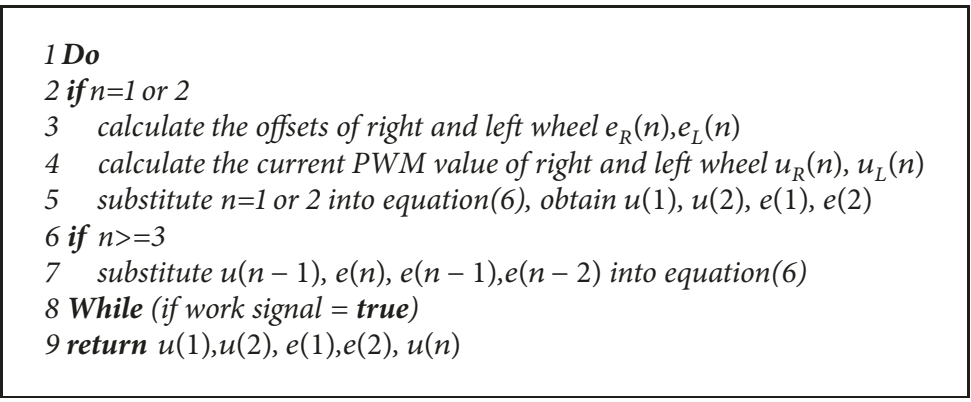

Algorithm 1: The implementation of PID algorithm.

[26], instead of calculating the forward speed of $V$ each wheel. Generally, there are two kinds of PID, as shown below.

$$
\begin{aligned}
& u(n) \\
& =K_{P}\left\{e(n)+\frac{T}{T_{I}} \sum_{i=0}^{n} e(i)+\frac{T_{D}}{T}[e(n)-e(n-1)]\right\} \\
& \quad+u_{0}
\end{aligned}
$$

Incremental PID control algorithm:

$$
\begin{aligned}
\Delta u(n)= & u(n)-u(n-1) \\
= & K_{P}[e(n)-e(n-1)]+K_{P} \frac{T}{T_{I}} e(n) \\
& +K_{P} \frac{T_{D}}{T}[e(n)-2 e(n-1)+e(n-2)] \\
e(n)= & r(t)-c(t) \\
c(t)= & N
\end{aligned}
$$

where $u(n)$ is the output value of PID and $r(t)$ is the desired value. $N$ is the photoelectric encoder current measurement value, and $e(n)$ is the deviation value. In this paper, DC motor is used for robotic motion, by controlling the duty rate of PWM to control the velocity, so according to the incremental PID control algorithm, the recursion formula is

$$
\begin{aligned}
u(n)= & u(n-1)+\Delta u(n) \\
= & u(n-1)+K_{P}\left(1+\frac{T}{T_{I}}+\frac{T_{D}}{T}\right) e(n) \\
& -K_{P}\left(1+2 \frac{T_{D}}{T}\right) e(n-1)-K_{P} e(n-2) \\
= & u(n-1)+b_{0} e(n)+b_{1} e(n-1)+b_{2} e(n-2) \\
b_{0}= & K_{P}\left(1+\frac{T}{T_{I}}+\frac{T_{D}}{T}\right) \\
b_{1}= & -K_{P}\left(1+2 \frac{T_{D}}{T}\right) \\
b_{2}= & -K_{P}
\end{aligned}
$$

where $u(n)$ is currently the value of PWM, which supplies motor; additionally, $u(n-1)$ is the last value of PWM. $e(n)$, $e(n-1), e(n-2)$ are the offsets between expected velocity and real velocity by current, by last time, and by the time before last time. $T$ is the sample time, usually a fixed value; $K_{P}, T_{I}, T_{D}$ are the parameters which will be optimized. The algorithm's pseudocode is as in Algorithm 1.

Among them, $u(1), u(2), e(1), e(2)$ are the PID output values and offsets of the left and right wheel, respectively. Using PID algorithm, the mechanical errors can be eliminated, and the robot can ensure that it is straight as much as possible.

\subsection{Positioning and Navigation Algorithm}

3.2.1. Angle and Position Correction. The two wheels of the sweeping robot are driven by two independent motors. Although using PID regulation control can ensure that the two wheels are at the same speed, the robot would shift when running a distance because there may exist slipping and mechanical error. A new method of angle and position correction based on infrared and directional ultrasonic sensors is presented in this paper as shown in Figure 5.

The navigator is placed in the middle of the wall on one side of the room, emitting vertical infrared ray and fanshaped ultrasonic waves on both sides. The angle of motion of the robot is always parallel to the wall. When the robot runs from top to bottom and from right to left, and each time is in the middle of the room, the robot receives infrared ray and ultrasonic waves, determining its position by combining onboard devices. If the robot discovers deviation from the straight line, it corrects itself in time.

Basic work principle: With the infrared ray by the navigator treated as the baseline, we sent an ultrasonic wave with certain angles in particular frequency which can measure the precise position of robot. Based on two times of measurement, we can resolve robot's deviation angle. Specific steps are as follows (before the algorithm is detailed, note that we briefly define the notation that is used throughout the paper in Table 1):

Step 1. When the robot arrives at the baseline of infrared, after it receives an infrared ray, the distance $\vec{a}$ between them can be obtained. 


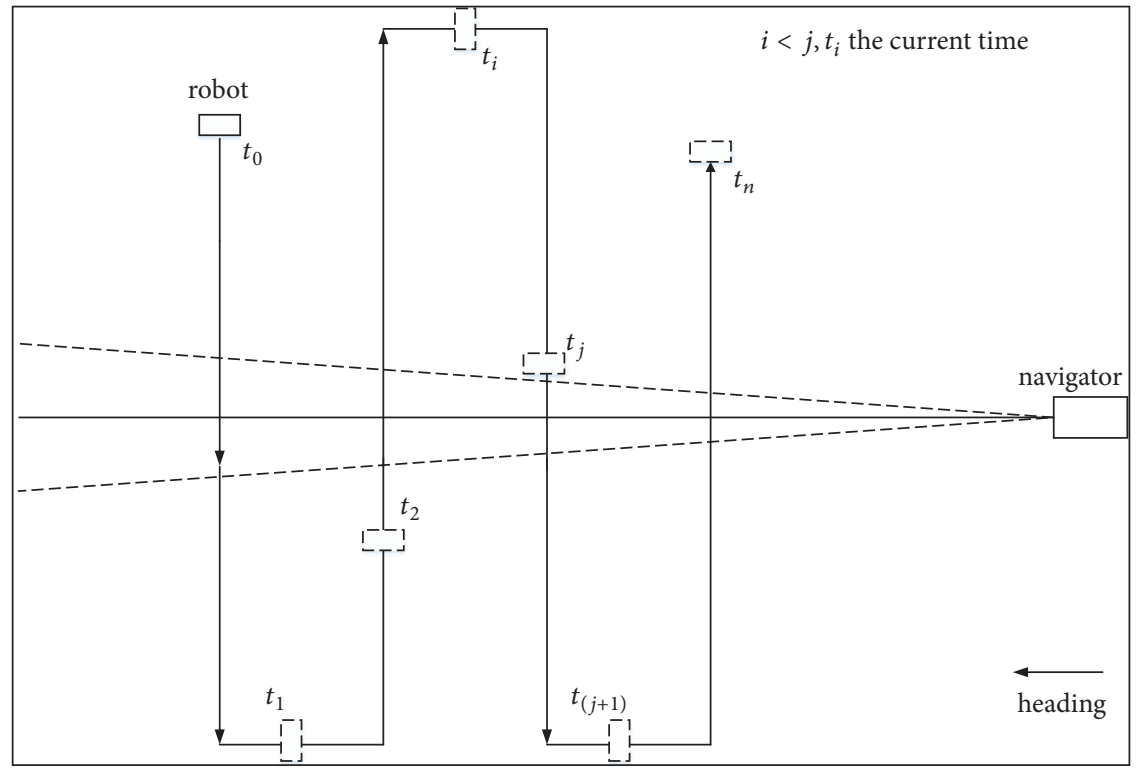

FIgURE 5: A diagram of the sweeping robot working $\mathrm{Ti}(\mathrm{i}=0, \ldots, \mathrm{n})$.

TABLE 1: The model of analytic geometry parameters.

\begin{tabular}{llll}
\hline Symbol & Description & Symbol & Description \\
\hline O & $\begin{array}{c}\text { The position of } \\
\text { navigator }\end{array}$ & $\begin{array}{c}\text { The Expected } \\
\text { position of robot }\end{array}$ \\
B & The real position of robot & The distance of O to A \\
$\mathrm{C}$ & The vector of O to A & $|\vec{a}|$ & The distance of O to B \\
$\vec{a}$ & The vector of O to B & $|\vec{b}|$ & The distance of A to B \\
$\vec{b}$ & The vector of A to B & $|\vec{c}|$ & The distance of O to C \\
$\vec{c}$ & The vector of O to C & $|\vec{d}|$ & The distance of A to C \\
$\vec{d}$ & The vector of A to C & $|\vec{e}|$ &
\end{tabular}

Step 2. After the robot leaves the baseline and runs in a short distance $|\vec{e}|$, measure the distance between them by ultrasonic wave again.

Step 3. Resolve the horizontal attitude angle $\alpha$ from the relationship of geometry.

$$
\alpha=\arccos \frac{|\vec{\alpha}|^{2}+|\vec{e}|^{2}-|\vec{d}|^{2}}{2|\vec{e}||\vec{a}|}
$$

However, as the complexity of the formula $\alpha$ is expensive and the gap between $\vec{e}$ and $\vec{a}$ is more than $10^{3}$ orders of magnitude, we propose a geometry algorithm to make the complexity reduction substantial (see Figure 6).

Follow $O$ as the center of a circle, the direction of $\vec{a}$ as $\mathrm{x}$ axis forward, and the direction of perpendicular $\vec{a}$ as y axis forward. Let down $\odot O$ trajectory is $y=f(x)$. The slope of the tangent at $\mathrm{C}$ is $f^{\prime}(c)$ in $\odot O$.
The slope of AC: because of $|\vec{b}|$ towards 0 relative to $\vec{a}$, $x_{C}-x_{A}=\mathrm{o}(\mathrm{x})$ [27], $\mathrm{o}(\mathrm{x})$ is the infinitely small quantity of $\mathrm{x}$.

$$
f^{\prime}\left(x_{C}\right)=\lim _{x_{C} \longrightarrow x_{A}} \frac{f\left(x_{C}\right)-f\left(x_{A}\right)}{x_{C}-x_{A}}
$$

so, the slope of the tangent at $\mathrm{C}$ being $f^{\prime}(c)$ in $\odot \mathrm{O}$ is equal to the slope of AC.

In the circle, from the relationship of geometry $\vec{d}$. $\left(1, f^{\prime}(c)\right)=0$, then $\vec{d} \cdot \vec{e}=0$.

Similarly, $\vec{a} \cdot \vec{e}=0$, from the relationship of geometry $\langle-\vec{a}, \vec{c}\rangle=\langle-\vec{a}, \vec{e}\rangle+\langle-\vec{e}, \vec{c}\rangle$

$$
\alpha=90^{\circ}+\beta=90^{\circ}+\arcsin \frac{|\vec{b}|-|\vec{a}|}{|\vec{c}|}
$$

As the (10) and (12) are antitrigonometric functions, we can directly consider the internal formula. As we all know, 


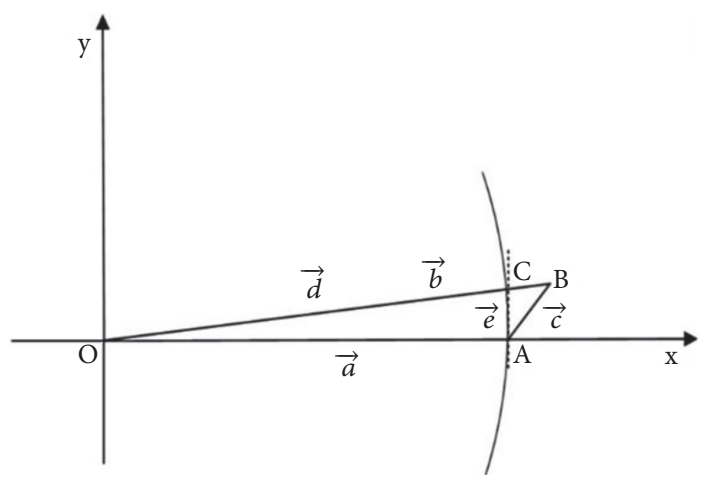

FIGURE 6: Establishing coordinate system for the motion model of robot.

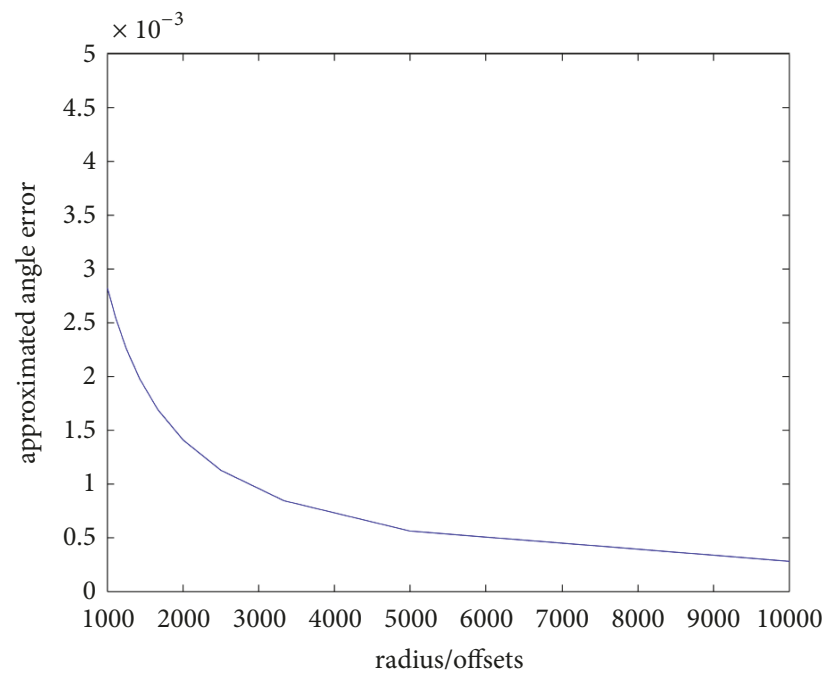

FIGURE 7: The relationship between radius/offsets and approximate angle error.

multiplication is nearly 10 times slower than addition and subtraction, and division is nearly 20 times slower than addition and subtraction, when it is an 8-bit CPU. Now, we define the time of addition and subtraction as $t$, then the multiplication is $10 t$, and division is $20 t$. For (10), the time of the whole equation is as follows.

$$
10 t+10 t+10 t+2 t+20 t+10 t+10 t=72 t
$$

For equation (12), the time of the whole equation is as follows.

$$
t+20 t=21 t
$$

Then, we have the following.

$$
\frac{72 t-21 t}{72 t} * 100 \%=70.8 \%
$$

Compared with (10), (12) reduces the computational complexity [28] and increases efficiency by nearly $70.8 \%$. At the same time, it satisfies the actual precision requirement under the condition of $\widehat{A C}$ being smaller. The relationship between relative errors and deviation angle from the original line is shown in Figure 7.
3.2.2. Navigation Implementation between Multiple Rooms. In order to help a robot to cross different rooms to work continuously, it should be improved autonomously. In this paper, we built topology model for multiple rooms based on the theory of graph optimization [29]. Meanwhile, the depthfirst search (DFS) [30] stack is introduced to firstly select the most nearest and inmost room to work [31], which improves the efficiency of DFS. The algorithm's pseudocode is as in Algorithm 2.

In this algorithm, we define a room as $V_{i}(i=1 \ldots n)$ by the navigator which has a unique encode signal. We build a stack to store the room information so that the robot can select the most nearest and inmost room to work. When it finishes the current room, then it checks the stack status every time. Lastly, it goes back to the initial room and stops working.

\section{Implementation and Experiments}

We tested the SLMN algorithm in a $10.8 * 10 \mathrm{~m}$ real indoor environment, and the dimension of the robot was $0.8 * 0.4 \mathrm{~m}$. Since it is an upgraded TF card, the robot could easily store the data from the electronic motor encoder. The real robot was shown in Figure 8. 


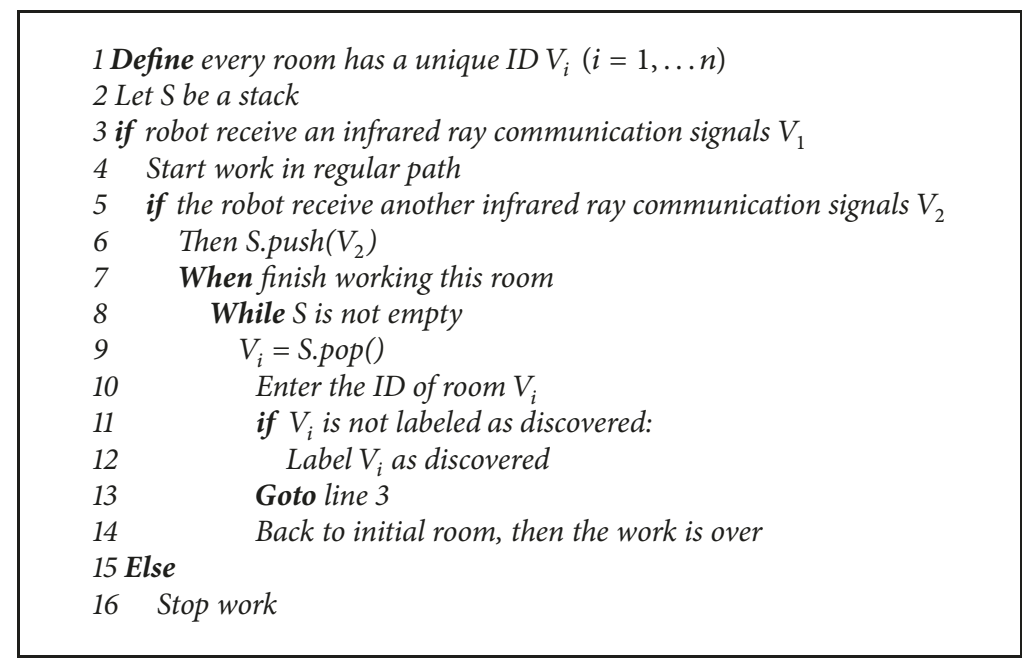

Algorithm 2: DFS stack for cleaning work.

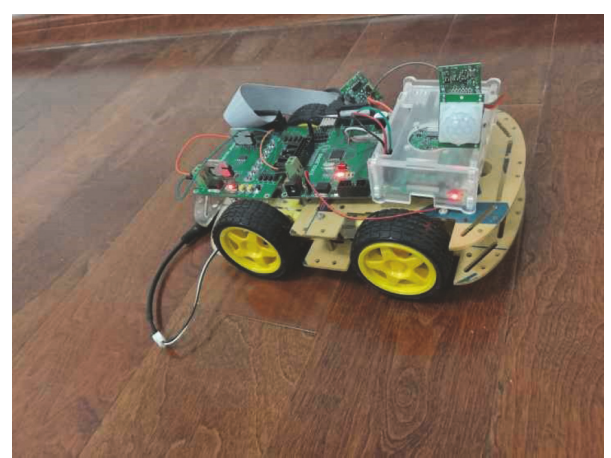

Figure 8: The model of a real robot.

After finishing its work run, the local trajectory of the robot was restored (as the errors are small, it is difficult to discover the trajectory of at a global view), as shown in Figure 9. The red and blue lines represent the robot's true trajectory and ideal trajectory, respectively. Therefore, we can find out that when the robot passed an infrared reference line $(y=5 \mathrm{~m})$ by the navigator, the deviation trajectory would be effectively corrected.

By calculating the absolute value, a good view of this deviation could be created. The deviation that appears on $[(5+10.4 k, 5+10.4(k+1)]$ will be effectively corrected at $5+10.4(k+1)$ as shown in Figure 10 .

Occasionally, resources such as memory and computational time are of primary concern, but most often cost and accuracy are what we want to measure. By analyzing several candidate algorithms for the navigation problem [6], we can identify a most efficient one. In this paper, we put the results of comparative analysis with other state-of-theart algorithms such as vSLAM [11], LIDAR SLAM [17], and inertial navigation $[32,33]$ as shown in Table 2.

From Table 2, it is seen that the two SLAM systems have similar precision, which are the most highest. However, their work time is also the shortest in the same battery capacity, so it needs more power to keep it working. Although inertial navigation system has the lowest cost, its error is too enormous to work normally. As a result, since the development of new energy still faces several uncertain difficulties [3436], our proposed algorithm will cost less energy in satisfying practical applications. which than the other state-of-the-art algorithms.

\section{Conclusion}

On the one hand, this paper proposed a high efficiency method on indoor sweep robot line navigation. The two measured values between navigator and robot and the distance of robot motion can form a triangle then to solve the forward angle and correct the direction of forward speed with analytic geometry principle. Finally, we implemented the long time line navigation, and based on that, we propose multiroom navigation algorithm. After simulation and reality environment experiments, on the threshold of error, an indoor line navigation method based on the navigator which consists of the infrared and ultrasonic sensors, even in the evening, could resolve the problems on the other existing navigation.

On the other hand, we prepare to do more works on building a map based on the high efficiency motion to 
TABLE 2: The result of performance test.

\begin{tabular}{|c|c|c|c|c|}
\hline \multirow{3}{*}{ Compared Algorithms } & \multicolumn{4}{|c|}{ Precise and cost } \\
\hline & \multirow{2}{*}{ Mean relative errors in $500 \mathrm{~m}^{2}(\%)$} & \multicolumn{3}{|c|}{ Battery life with $12 \mathrm{~V}(h)$} \\
\hline & & $1 A H$ & $3 A H$ & $5 A H$ \\
\hline Proposed & 6.73 & 1.7 & 4.9 & 7.6 \\
\hline vSLAM & 2.32 & 1.4 & 4.1 & 6.3 \\
\hline Radar SLAM & 3.17 & 1.3 & 3.9 & 5.9 \\
\hline Inertial navigation & 13.6 & 2.1 & 6.1 & 9.8 \\
\hline
\end{tabular}

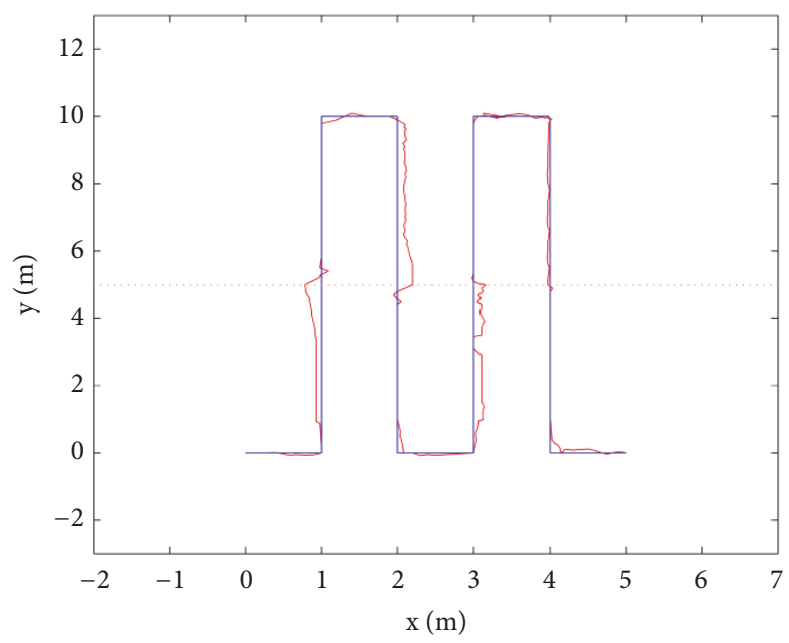

Figure 9: The local trajectory of the robot had been restored, a red line is the real trajectory of the robot, and a blue line is the expected trajectory of the robot.

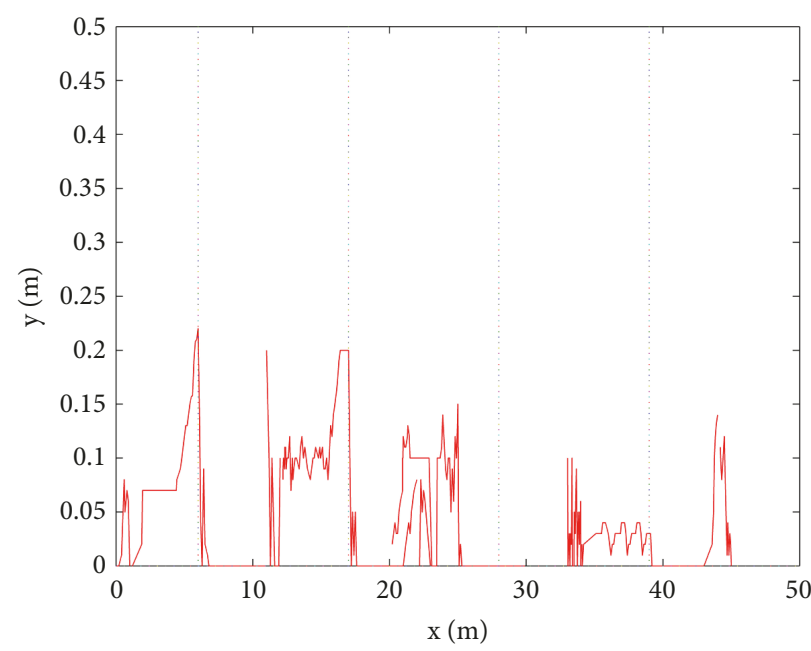

FIGURE 10: The variation trend of the deviation.

improve its practicability and integrity, so it can become selfcharging and work continuously.

\section{Data Availability}

The data used to support the findings of this study are available from the corresponding author upon request.

\section{Conflicts of Interest}

The authors declare that they have no conflicts of interest.

\section{Acknowledgments}

This work was supported by National Key R\&D Program of China Nos. 2018YFC0806900, 2016YFC0800606, 2016 
YFC0800310, and 2018YFC0407905; Natural Science Foundation of Jiangsu Province under Grants Nos. BK20150721 and BK20161469; and Primary Research \& Development Plan of Jiangsu Province under Grants Nos. BE2015728, BE2016904, BE2017616, and BE2018754.

\section{References}

[1] L. Jia-jun, Z. Bi, and H. Yuan-lie, "Research on Path Planning Algorithm for Cleaning Robot Based on Improved Potential Field Grid Method," Journal of Guangdong University of Technology, 2016.

[2] J. Shang, X. Hu, F. Gu, D. Wang, and S. Yu, "Improvement schemes for indoor mobile location estimation: a survey," Mathematical Problems in Engineering, vol. 2015, Article ID 397298, 32 pages, 2015.

[3] G. Liu, Y. Geng, and K. Pahlavan, "Effects of calibration RFID tags on performance of inertial navigation in indoor environment," in Proceedings of the 2015 International Conference on Computing, Networking and Communications, ICNC 2015, pp. 945-949, USA, February 2015.

[4] B.-W. Li and D.-Y. Yao, "Low-cost MEMS IMU navigation positioning method for land vehicle," Zhongguo Guanxing Jishu Xuebao/Journal of Chinese Inertial Technology, vol. 22, no. 6, pp. 719-723, 2014.

[5] S. Lynen, M. W. Achtelik, S. Weiss, M. Chli, and R. Siegwart, "A robust and modular multi-sensor fusion approach applied to MAV navigation," in Proceedings of the 2013 26th IEEE/RSJ International Conference on Intelligent Robots and Systems: New Horizon, IROS 2013, pp. 3923-3929, Japan, November 2013.

[6] Y. Zheng, G. Shen, L. Li, C. Zhao, M. Li, and F. Zhao, “TraviNavi: self-deployable indoor navigation system," IEEE/ACM Transactions on Networking, no. 99, pp. 1-15, 2014.

[7] J. Rodas, C. J. Escudero, and D. I. Iglesia, "Bayesian filtering for a Bluetooth positioning system," in Proceedings of the 2008 IEEE International Symposium on Wireless Communication Systems, ISWCS'08, pp. 618-622, Iceland, October 2008.

[8] P. Lin, Q. Li, Q. Fan, X. Gao, and S. Hu, "A real-time locationbased services system using WiFi fingerprinting algorithm for safety risk assessment of workers in tunnels," Mathematical Problems in Engineering, vol. 2014, Article ID 371456, 10 pages, 2014.

[9] J. Fuentes-Pacheco, J. Ruiz-Ascencio, and J. M. RendónMancha, "Visual simultaneous localization and mapping: a survey," Artificial Intelligence Review, vol. 43, no. 1, pp. 55-81, 2015.

[10] S. Leutenegger, S. Lynen, M. Bosse, R. Siegwart, and P. Furgale, "Keyframe-based visual-inertial odometry using nonlinear optimization," International Journal of Robotics Research, vol. 34, no. 3, pp. 314-334, 2015.

[11] R. Mur-Artal and J. D. Tardos, "ORB-SLAM2: An Open-Source SLAM System for Monocular, Stereo, and RGB-D Cameras," IEEE Transactions on Robotics, vol. 33, no. 5, pp. 1255-1262, 2017.

[12] S. Yin, X. Li, H. Gao, and O. Kaynak, "Data-based techniques focused on modern industry: an overview," IEEE Transactions on Industrial Electronics, vol. PP, no. 99, p. 1, 2014.

[13] R. A. Newcombe, S. J. Lovegrove, and A. J. Davison, "DTAM: Dense tracking and mapping in real-time," in Proceedings of the 2011 IEEE International Conference on Computer Vision, ICCV 2011, pp. 2320-2327, Spain, November 2011.
[14] J. Smisek, M. Jancosek, and T. Pajdla, "3D with Kinect, Advances in Computer Vision Pattern Recognition," 3D with Kinect, Advances in Computer Vision Pattern Recognition, vol. 21, no. 5, pp. 1154-1160, 2013.

[15] P. K. Binu, R. A. Krishnan, and A. P. Kumar, "An efficient indoor location tracking and navigation system using simple magnetic map matching," in Proceedings of the 2016 IEEE International Conference on Computational Intelligence and Computing Research (ICCIC), pp. 1-7, Chennai, December 2016.

[16] A. Sud, R. Gayle, E. Andersen, S. Guy, M. Lin, and D. Manocha, "Real-time navigation of independent agents using adaptive roadmaps," in Proceedings of the ACM SIGGRAPH 2008 Classes, 2015.

[17] W. Hess, D. Kohler, H. Rapp, and D. Andor, "Real-time loop closure in 2D LIDAR SLAM," in Proceedings of the 2016 IEEE International Conference on Robotics and Automation, ICRA 2016, pp. 1271-1278, Sweden, May 2016.

[18] K. Wang, T. Song, T. Liang et al., "A dual-infrared-transmitter optical wireless based indoor user localization system with high accuracy," in Proceedings of the 2017 Optical Fiber Communications Conference and Exhibition, OFC 2017, USA, March 2017.

[19] J. Krejsa and S. Vechet, "Infrared beacons based localization of mobile robot," Elektronika ir Elektrotechnika, no. 1, pp. 17-22, 2012.

[20] J. Yun and M.-H. Song, "Detecting direction of movement using pyroelectric infrared sensors," IEEE Sensors Journal, vol. 14, no. 5, pp. 1482-1489, 2014.

[21] D. Han, S. Jung, M. Lee, and G. Yoon, "Building a practical wifi-based indoor navigation system," IEEE Pervasive Computing, vol. 13, no. 2, pp. 72-79, 2014.

[22] C. Yang and H.-R. Shao, "WiFi-based indoor positioning," IEEE Communications Magazine, vol. 53, no. 3, pp. 150-157, 2015.

[23] P. Shah and S. Agashe, "Review of fractional PID controller," Mechatronics, vol. 38, pp. 29-41, 2016.

[24] R. Mur-Artal, J. M. M. Montiel, and J. D. Tardos, "ORBSLAM: a versatile and accurate monocular SLAM system," IEEE Transactions on Robotics, vol. 31, no. 5, pp. 1147-1163, 2015.

[25] J. Simanek, M. Reinstein, and V. Kubelka, "Evaluation of the EKF-based estimation architectures for data fusion in mobile robots," IEEE/ASME Transactions on Mechatronics, vol. 20, no. 2, pp. 985-990, 2015.

[26] K. J. Astrom, PID Controllers: Theory, Design and Tuning, Instrument Society of America Research Triangle Park Nc, 1995.

[27] A. Banner, “The Calculus Lifesaver," 2009.

[28] T. Hcormen, C. Eleiserson, R. Lrivest, and C. Stein, Introduction to Algorithms, vol. 39, Computer Education, 3rd edition, 2013.

[29] S. R. U. Murty, Graph Theory with Applications: North Holland, 1976.

[30] K. Mehlhorn, "Engineering DFS-based graph algorithms," 2017, https://arxiv.org/abs/1710.06055.

[31] R. Tarjan, "Depth-first search and linear graph algorithms," in Proceedings of the 12th Annual Symposium on Switching and Automata Theory (swat 1971), pp. 114-121, East Lansing, MI, USA, October 1971.

[32] A. R. Jimenez, F. Seco, J. C. Prieto, and J. Guevara, "Indoor Pedestrian navigation using an INS/EKF framework for yaw drift reduction and a foot-mounted IMU," in Proceedings of the 2010 7th Workshop on Positioning, Navigation and Communication, WPNC'10, pp. 135-143, Germany, March 2010.

[33] B. Barshan and H. F. Durrant-Whyte, "Inertial navigation systems for mobile robots," IEEE Transactions on Robotics and Automation, vol. 11, no. 3, pp. 328-342, 1998. 
[34] K. Wang, J. Pang, L. Li, S. Zhou, Y. Li, and T. Zhang, "Synthesis of hydrophobic carbon nanotubes/reduced graphene oxide composite films by flash light irradiation," Frontiers of Chemical Science and Engineering, vol. 12, no. 3, pp. 376-382, 2018.

[35] W. Kai, L. Liwei, X. Wen et al., "Electrodeposition synthesis of PANI/MnO2/graphene composite materials and its electrochemical performance," International Journal of Electrochemical Science, vol. 12, no. 9, pp. 8306-8314, 2017.

[36] W. Kai, "Synthesis of porous carbon by activation method and its electrochemical performance," International Journal of Electrochemical Science, pp. 10766-10773, 2018. 


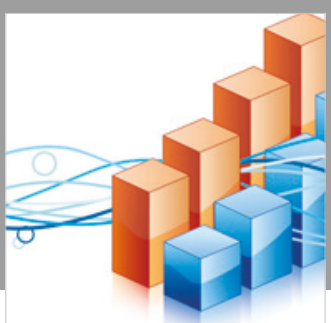

Advances in

Operations Research

\section{-n-m}
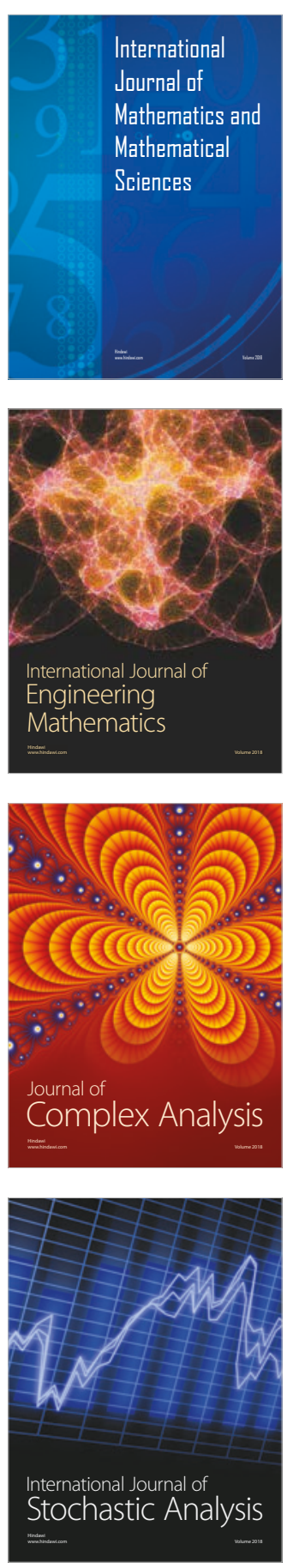
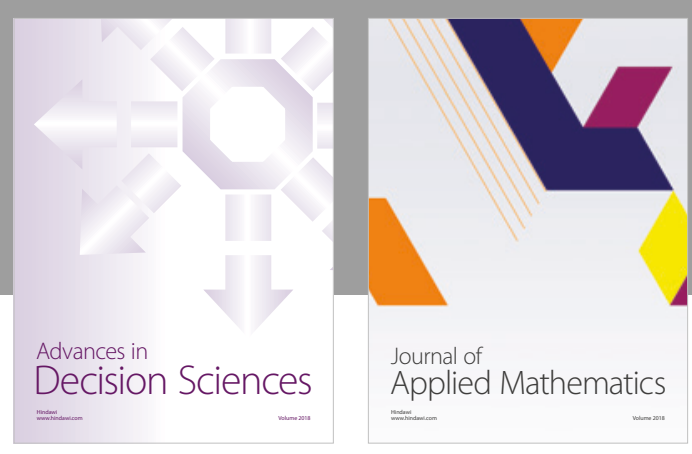

Journal of

Applied Mathematics
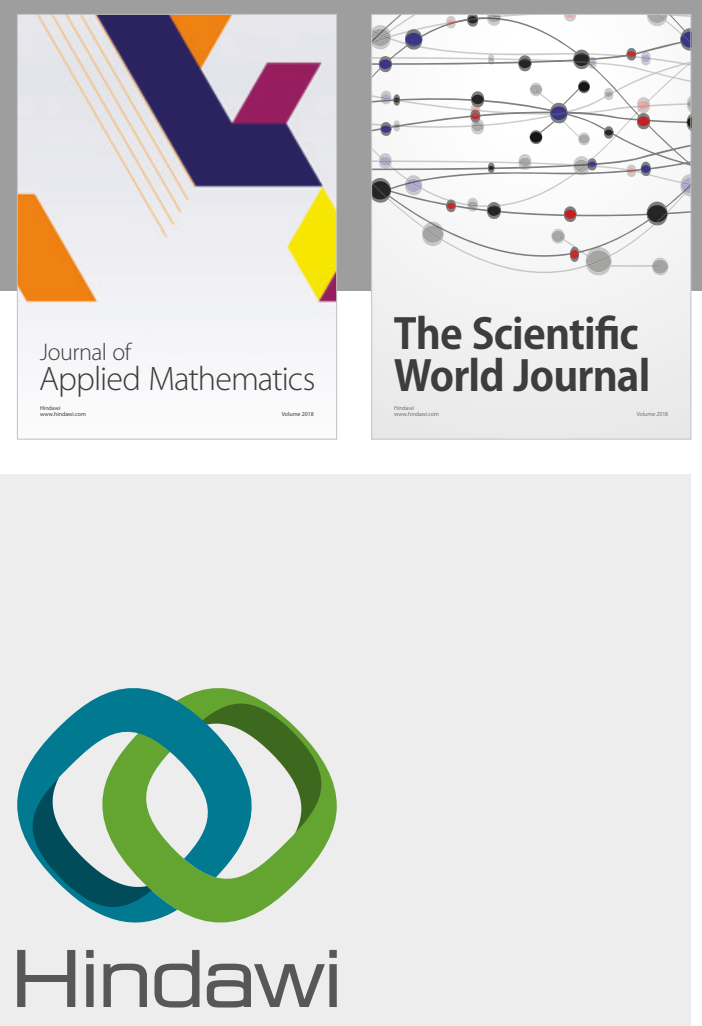

Submit your manuscripts at

www.hindawi.com

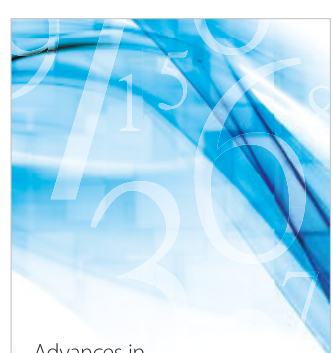

Advances in
Numerical Analysis
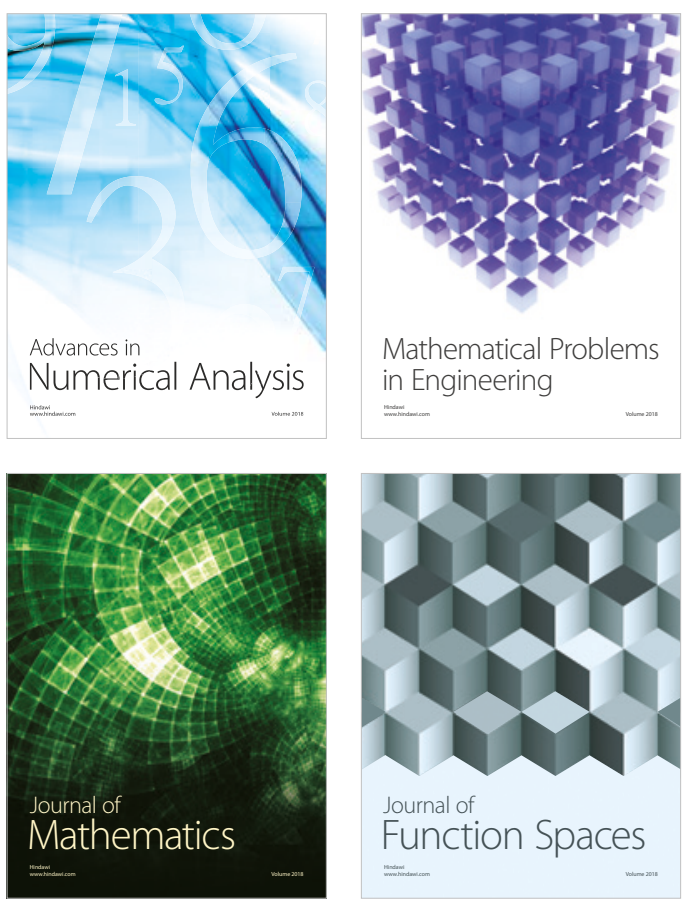

Mathematical Problems in Engineering

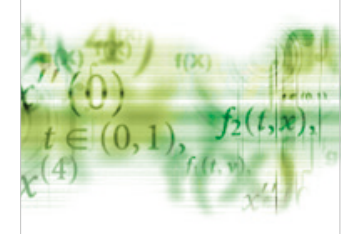

International Journal of

Differential Equations

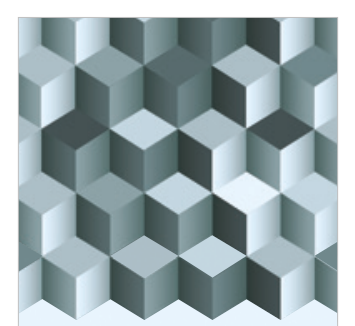

Journal of

Function Spaces

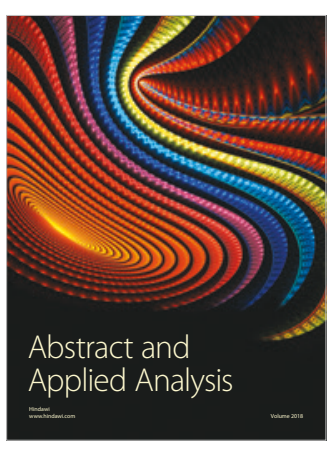

The Scientific

World Journal

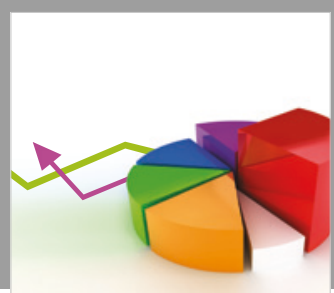

Journal of

Probability and Statistics
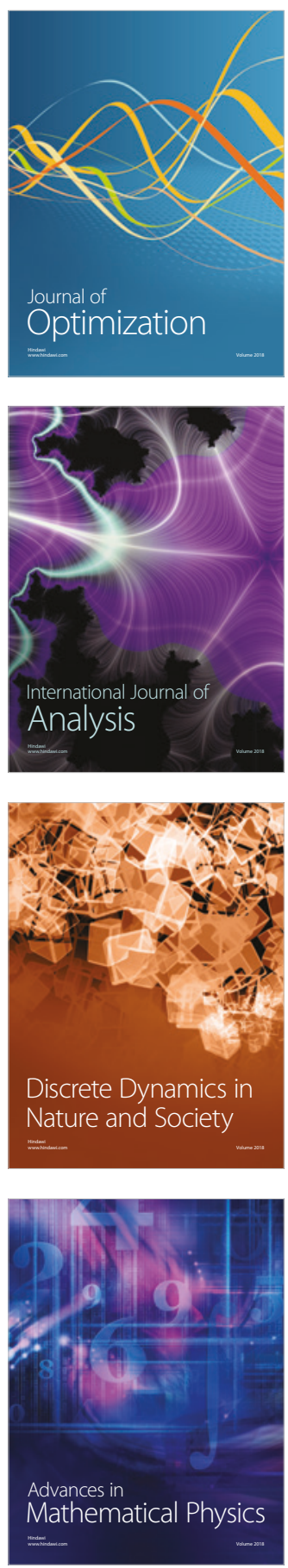